\title{
Screening for abdominal aortic aneurysm
}

\author{
Remains effective and is likely to be cost effective in the UK, but data \\ need to be monitored
}

\section{RESEARCH, pp 1538, 1542}

\section{Martin J Buxton professor} of health economics, Health Economics Research Group Brunel University, Uxbridge UB83PH

martin.buxton@brunel.ac.uk Provenance and peer review: Commissioned; not externally peer reviewed

Cite this as: BMJ 2009;338:b2185 doi: 10.1136/bmj.b2185
A national NHS programme in England offers screening for abdominal aortic aneurysm in men aged 65 , with the aim of reducing deaths from aneurysm rupture. ${ }^{1}$ The key evidence underpinning this policy was the results of the multicentre aneurysm screening study (MASS), funded by the Medical Research Council. ${ }^{2}$ More than 67000 men aged $65-74$ were recruited from four centres in the UK and were randomised to either receive or not receive an invitation to screening. The results after 4 years' follow-up showed a $42 \%$ relative risk reduction in mortality related to abdominal aortic aneurysm (absolute risk reduction from $0.33 \%$ to $0.19 \%$ ). An economic evaluation indicated that the investment in screening was close to being acceptably cost effective, even in the short term. ${ }^{3}$

The evidence of clinical effectiveness was reinforced by a subsequent Cochrane Review-which estimated a $40 \%$ relative risk reduction (absolute risk reduction from $0.27 \%$ to $0.16 \%$ )-and then by results from MASS after 7 years' follow-up. ${ }^{45} \mathrm{~A}$ formal long term economic model built on the MASS data indicated that abdominal aortic aneurysm screening was likely to be extremely cost effective. ${ }^{6}$ Taken together, these findings provided an unusually solid evidence base for a screening policy.

Two linked studies further add to the evidence base. ${ }^{78}$ In the first study, Thompson and colleagues provide 10 year follow-up results from MASS, in which the relative risk reduction in mortality related to abdominal aortic aneurysm is maintained. ${ }^{7}$ They recalculate the original cost effectiveness estimates to account for this extended period of observation and now indicate an incremental cost effectiveness ratio of $£ 7600$ (€8800, \$12500; 95\% CI £5100 to £13000 (€5900 to $€ 15000, \$ 8350$ to $\$ 21300$ ) per life year gained, much lower than the $£ 20000-30000$ threshold used by the National Institute for Health and Clinical Evidence (NICE). On the other hand, the second linked study by Ehlers and colleagues presents a lifetime modelling study of a screening programme for abdominal aortic aneurysm and concludes that screening is not cost effective, with a mean cost per quality adjusted life year of $£ 43485 .{ }^{8}$ What is the reason for this discrepancy, particularly given that the modelling paper uses the previously published cost effectiveness evidence from MASS?

From the evidence in the papers as presented here, it is hard to identify the key reason for the differing findings or, more likely, the most important of multiple contributory reasons. Importantly, the two papers agree that screening significantly reduces the number of deaths related to abdominal aortic aneurysm in the long term, although the Danish model suggests that there may be a small increase in net deaths in the short term. Ehlers and colleagues also argue, as have others, ${ }^{9}$ that a model that draws on all available data is inherently superior to a model based around one trial, albeit the biggest. Ehlers and colleagues have directly modelled the effects of the current policy of screening 65 year olds, whereas the MASS results reflect a sample group screened at age 65-74.

The Danish authors do recognise, however, that there are other important differences between their long term cost effectiveness model and cost effectiveness estimates based directly on MASS, including their use of Danish data on long term mortality after elective and emergency surgery. Possibly the key difference is the cost data used. Ehlers and colleagues used estimates of Danish costs that differed in important ways from the estimates originally in MASS-the costs of screening are higher in Denmark compared with the UK and the differential cost between elective and emergency surgery is substantially lower. Both these factors would make screening seem less cost effective. This is just one difference between the models, however, and cost effectiveness ratios are known to be relatively unstable where the average difference in effect is small (at 10 years, an average additional survival of just 4.8 days per man invited to screening according to the MASS data).

Understanding fully the differences between modelling

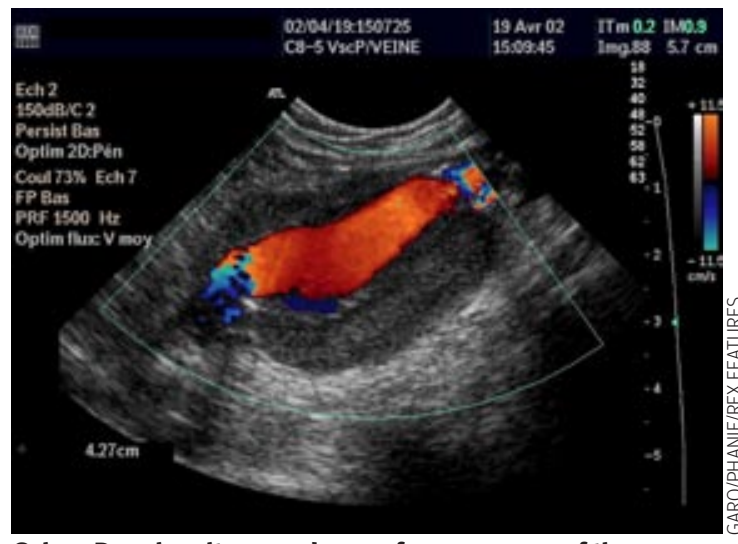

Colour Doppler ultrasound scan of an aneurysm of the abdominal aorta showing circulating lumen of the artery (orange); the rest of the bulge is occupied by a clot (dark grey) 
studies usually requires access to and exploration of the models-for example, by substituting alternative data and assumptions, and, most importantly in this case, incorporating into each the new 10 year MASS results. The need to explore what happens when parameter estimates and model structures are changed is why NICE insists on access to a transparent working version of models when appraising new technologies. A compromise for journals would be to insist that much more detailed explanations of the models that underpin articles are made available on the internet.

So without such additional information and analyses, what should we conclude about the cost effectiveness of screening for abdominal aortic aneurysm? The 10 year results from MASS confirm that screening is effective in the long term, and there is no indication of the survival benefit being eroded. It would also seem reasonable to infer that a UK screening programme will be acceptably cost effective, providing that effectiveness can be maintained across the country and that the cost estimates remain relevant. These caveats emphasise that screening costs need to be controlled while quality is maintained, and that changes in the management of elective or emergency repair of aneurysms might have a significant impact. For example, as indicated in one of the sensitivity analyses in the Danish paper, the introduction of more costly endovascular repair might reduce the cost effectiveness of screening. ${ }^{9}$
The accumulated evidence suggests that a national screening programme in the UK is appropriate and likely to be cost effective, but its costs and outcomes need to be carefully monitored and the data need to be regularly re-analysed to ensure that both the effectiveness and cost effectiveness remain acceptable in the context of changing practice.

1 National Screening Committee. National Screening Committee policy-abdominal aortic aneurysm screening. February 2009. http:// www.library.nhs.uk/screening/ViewResource.aspx?resID=60457 (accessed 27 May 2009).

2 Multicentre Aneurysm Screening Study Group. The Multicentre Aneurysm Screening Study (MASS) into the effect of abdominal aortic aneurysm screening on mortality in men: a randomised controlled trial. Lancet 2002;360:1531-9.

3 Multicentre Aneurysm Screening Study Group. Multicentre aneurysm screening study (MASS): cost effectiveness analysis of screening for abdominal aortic aneurysms based on four year results from randomised controlled trial. BMJ 2002;325:1135-8.

4 Cosford PA, Leng GC. Screening for abdominal aortic aneurysm. Cochrane Database Syst Rev 2007;(2):CD002945. doi:1002/14651858.CD002945.pub2.

5 Kim LG, Scott RAP, Ashton HA, Thompson SG. A sustained mortality benefit from screening for abdominal aortic aneurysm. Ann Intern Med 2007;146:699-706.

6 Kim LG, Thompson SG, Briggs AH, Buxton MJ, Campbell HE. How cost-effective is screening for abdominal aortic aneurysms? J Med Screening 2007;14:46-52.

7 Thompson SG, Ashton HA, Gao L, Scott RAP. Screening men aged 65 or more for abdominal aortic aneurysm: 10 year mortality and cost effectiveness results from the Multicentre Aneurysm Screening Study. BM/ 2009.338:b2307.

8 Ehlers L, Overvad K, Sørensen J, Christensen S, Bech M, Kjølby M. Analysis of cost effectiveness of screening Danish men aged 65 for abdominal aortic aneurysm. BMJ 2009;338:b2243.

9 Sculpher MJ, Claxton K, Drummond M, McCabe C. Whither trial-based economic evaluation for health care decision making? Health Econ 2006;15:677-87.

\section{Smoking in pregnancy}

\section{Severe adverse effects can be avoided if smoking is stopped early}

\section{RESEARCH, p 1552}

Rona Campbell professor

of health services research,

Department of Social Medicine,

University of Bristol, Bristol

BS82PS

rona.campbell@bris.ac.uk

Deirdre J Murphy professor

of obstetrics, Department of

Obstetrics and Gynaecology,

Trinity College Dublin and Coombe

Women and Infants University

Hospital, Dublin 8, Ireland

Competing interests: None declared.

Provenance and peer review: Commissioned; not externally peer reviewed.

Cite this as: $B M J$ 2009;338:b2188 doi: $10.1136 /$ bmj.b2188
The harm to infants of in utero exposure to tobacco smoke from maternal smoking is well established, ${ }^{12}$ and a quarter of a century has passed since it was first shown in a clinical trial that stopping smoking during pregnancy could avoid the adverse effects of smoking on birth weight. ${ }^{3}$ The findings from the linked cohort study by McCowan and colleagues provide new observational evidence suggesting that for healthy women having their first baby, quitting smoking early in pregnancy (before 15 weeks' gestation) can significantly reduce rates of spontaneous preterm birth, small for gestational age, and complicated pregnancies compared to those of non-smokers. ${ }^{4}$ Continuing to smoke was associated with an almost threefold increase in the rate of spontaneous preterm birth $(4 \%, 4 \%$, and $10 \%$ for non-smokers, stopped smokers, and current smokers, respectively) and a nearly twofold increase in the rate of small for gestational age infants $(10 \%, 10 \%$, and $17 \%$, respectively).

In the United States, up to $40 \%$ of women quit smoking spontaneously before their first antenatal visit. ${ }^{256}$ For the remainder, maximising the opportunity to prevent the undesirable effects of smoking on outcomes in late pregnancy requires that maternity services have early contact with pregnant women. Recent guidelines from the National Institute for Health and Clinical Excellence (NICE) in the United Kingdom already suggest that women be booked for antenatal care ideally by 10 weeks' gestation. ${ }^{7}$

Although there is good evidence from a systematic review that interventions in pregnancy can reduce maternal smoking, cluster randomised trials of midwife led interventions have not shown effectiveness. This disappointing finding is most likely because of the reservations midwives have about introducing the subject of stopping smoking in early antenatal visits, when they are trying to establish a positive relationship with women. ${ }^{28}$ Being able to congratulate selfmotivated women who have ceased smoking by 15 weeks' gestation is one thing, but it is another task again to persuade continuing smokers of the benefits of smoking cessation.

We agree with McCowan and colleagues that encouraging women to quit smoking early in pregnancy should not be at the expense of efforts to assist women to stop smoking at any time during pregnancy. But we would go further and emphasise that reducing fetal and infant exposure to the harmful effects of tobacco 


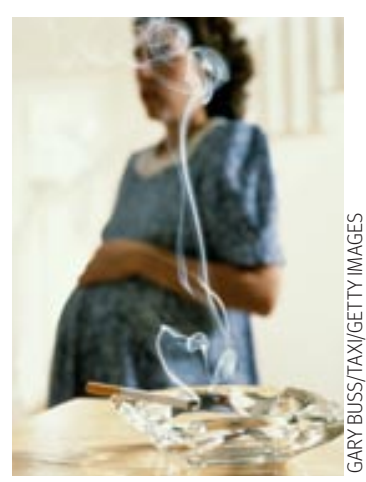

smoke requires health professionals to encourage and assist both parents and other adults to cease smoking, and every opportunity should be taken to do this.

There is ample evidence that brief physician advice to stop smoking increases cessation rates by $1-3 \%$, and further evidence indicates that more intensive counselling in healthcare settings will enable parents to stop smoking. ${ }^{910}$ Stopping parents smoking will reduce adverse pregnancy outcomes and improve their own health as well as that of their children. Given that parental smoking has been shown across the world to be the strongest predictor of children's smoking, reducing parental smoking will also diminish the uptake of smoking in adolescence. General practitioners, obstetricians, and midwives can also reinforce strategies for smoking control in their communities to reduce the initiation of smoking by young people. The National Institute for Health and Clinical Excellence has recently published guidance on mass media and point of sale measures for smoking cessation, and further guidance on school based interventions is to be published shortly. ${ }^{11}$

Persuading women to seek early antenatal careparticularly those who are socioeconomically disadvantaged and hence most likely to be smoking-will not be easy. Exactly how compelling the evidence is from the cohort study of McCowan and colleagues, and whether the evidence justifies the resources necessary to persuade women to seek early antenatal care should be questioned. The findings appear to be consistent with much earlier work showing that perinatal mortality and mean birth weight for babies born to women who stopped smoking by four months' gestation were almost the same as those for women who had never smoked. ${ }^{12}$ Smoking status in early pregnancy was the main exposure variable in this study, yet the study relied on self-reported data without biochemical verification, even though there is strong evidence from recent intervention trials that this method of data collection is subject to important reporting bias. ${ }^{2}$

There is also the potential for residual confounding in McCowan and colleagues' study, given the marked differences in social, demographic, and obstetric characteristics between the non-smokers, stopped smokers, and current smokers, although logistic regression analyses were performed. The data on pre-eclampsia rates would also have been useful, given the well established although counter-intuitive association between smoking and a reduced risk of pre-eclampsia.

From a research perspective, these findings need verification both observationally using data from other birth cohort studies and empirically, ideally through trials of interventions to assist smoking cessation early in pregnancy that have spontaneous preterm birth and small for gestational age as the primary outcomes. In the meantime, there are plenty of evidence based steps that clinicians can take, which could contribute to a reduction in the involuntary exposure of nearly half the world's children to harmful tobacco smoke. ${ }^{1}$

1 World Health Organization. International Consultation on Environmental Tobacco Smoke (ETS) and Child Health. Geneva: WHO, 2009. http:// www.who.int/tobacco/health impact/youth/ets/en/print.html.

2 LumleyJ, Oliver S, Chamberlain C, Oakley L. Interventions for promoting smoking cessation during pregnancy. Cochrane Database Syst Rev 2004;(4):CD001055. doi: 10.1002/14651858.CD001055.pub2.

3 Sexton M, Hebel JR. A clinical trial of change in maternal smoking and its effect on birth weight. JAMA 1984;251:911-5.

4 McCowan LME, Dekker GA, Chan E, Stewart A, Chappell LL, Hunter M, et al. Spontaneous preterm birth and small for gestational age infants in women who cease smoking in pregnancy: a prospective cohort study. BMJ 2009;338:b1081.

5 Quinn VP, Mullen PD, Ershoff DH. Women who stop smoking spontaneously prior to prenatal care and predictors of relapse before delivery. Addict Behav 1991;16:29-40.

6 Woodby LL,Windsor RA, Snyder SW, Kohler CL, Diclemente CC. Predictors of smoking cessation during pregnancy. Addiction 1999;94:283-92.

7 National Institute for Health and Clinical Excellence. Antenatal care: routine care for the healthy pregnant woman. NICE clinical guideline 62. London: NICE, 2008.

8 Moore L, Campbell R, Whelan A, Mills N, Lupton P, Misselbrook L, et al. Self help smoking cessation in pregnancy: cluster randomised controlled trial. $B M / 2002 ; 325: 1383-6$.

9 Stead LF, Bergson G, Lancaster T. Physician advice for smoking cessation. Cochrane Database Syst Rev2008;(2):CD000165. doi: 10.1002/14651858.CD000165.pub3.

10 Priest N, Roseby R, Waters E, Polnay A, Campbell R, Spencer N, et al. Family and carer smoking control programmes for reducing children's exposure to environmental tobacco smoke. Cochrane Database Syst Rev 2008:(4):CD001746. doi: 10.1002/14651858.CD001746.pub2.

11 National Institute for Health and Clinical Excellence. Mass-media and point-of-sales measures to prevent the uptake of smoking by children and young people. NICE public health guidance 14. London: NICE, 2008.

12 Butler NR, Goldstein H, Ross EM. Cigarette smoking in pregnancy: its influence on birth weight and perinatal mortality. BMJ 1972;2:127-30.

\title{
Monitoring bone mineral density during antiresorptive treatment for osteoporosis
}

\author{
Is potentially misleading and a misuse of healthcare resources
}

\section{RESEARCH, p 1553}

Juliet Compston professor of bone medicine jec1001@cam.ac.uk Competing interests: None declared. Provenance and peer review: Commissioned; not externally peer reviewed.

Cite this as: BMJ 2009;338:b1276 doi: 10.1136/bmi.b1276
Antiresorptive treatment for osteoporosis is usually prescribed for a minimum of five years. Although it reduces the risk of fracture, it does not relieve symptoms caused by existing fractures and may have side effects. Understandably, therefore, patients and their doctors seek reassurance that the treatment is working. The most common way to monitor response is repeated measurement of bone mineral density using dual energy $\mathrm{x}$ ray absorptiometry (DXA), an approach endorsed by recent guidelines. ${ }^{12}$ Routine monitoring is costly, however, and-if it does not reduce disease burden-may divert healthcare resources away from more deserving causes. In the linked study, Bell and colleagues assess the need to monitor the response to bisphosphonate treatment by estimating how much the effects of alendronate differ between individuals. ${ }^{3}$

Treatment aims to reduce the rate of fractures; therefore, monitoring should detect whether treatment will 


\section{A patient being prepared for a densitometry scan}

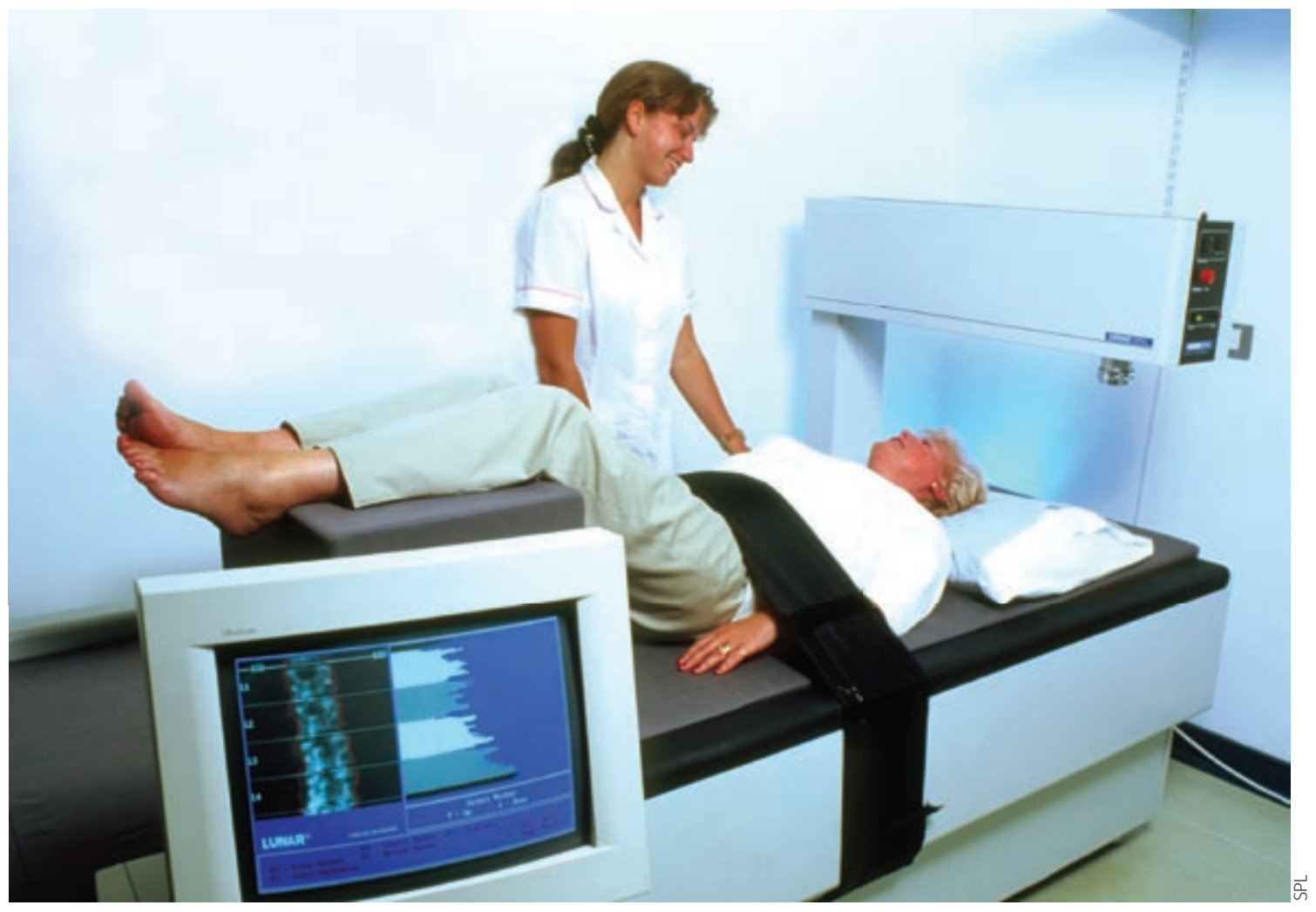

reduce the risk of fracture in individual patients. The effectiveness of repeated measurement of bone mineral density to monitor treatment depends on two prerequisites. Firstly, the test used (DXA) should be able to detect a significant change in bone mineral density within a time scale that enables effective changes in management to occur, and, secondly, the change in bone mineral density must predict the reduction in fractures after treatment. If these criteria are not satisfied, patients may be misled and receive inappropriate advice.

To detect significant changes in bone mineral density, the rate of bone gain must be larger than the precision error of the DXA measurement. Although this may be achieved after five years of bisphosphonate or other antiresorptive drugs, the change in bone mineral density within the first one or two years (when monitoring is recommended by some guidelines) is generally too small to be detected. In everyday clinical practice, the precision of bone mineral density measurements is likely to be worse than in clinical trials, and even changes of $7 \%$ or more may not be reliably shown in individual patients. ${ }^{4}$ Not being able to detect a change until five years after the start of treatment is clearly not clinically useful; even one year is too long given the high rate of recurrent fractures in the first 12 months after an incident fracture. ${ }^{5}$

The study by Bell and colleagues analyses changes in bone mineral density during treatment with alendronate in a large randomised controlled trial, and it strengthens the case against routine monitoring during the first few years of treatment. ${ }^{3}$ Around $97.5 \%$ of women treated with alendronate showed an increase in hip bone mineral density of at least $0.019 \mathrm{~g} / \mathrm{cm}^{2}$ after three years, and the variation in the effect of treatment between women was considerably less than the within person variation on treatment. In other words, although nearly all women gain bone mineral density during treatment, the large variability associated with the measurement of bone mineral density obscures the true treatment response in the individual, which makes monitoring of bone mineral density unnecessary and potentially misleading.

The final nail in the coffin for monitoring bone mineral density is the observation that only a small proportion of reduction in fractures attributable to treatment is explained by a change in bone mineral density. For of fracture associated with treatment with alendronate or raloxifene, respectively, is attributable to an increase in bone mineral density. ${ }^{67}$ Furthermore, some studies have found similar reductions in fracture regardless of whether bone mineral density increased or decreased on treatment. In postmenopausal women treated with risedronate, the reduction in non-vertebral fractures after three years of treatment was similar in women who lost or gained bone mineral density. ${ }^{8}$ Similar findings have been reported for the reduction in vertebral fractures in women treated with alendronate. ${ }^{9}$ In the MORE study of raloxifene treatment, women in the treatment group with $4 \%$ bone loss in the hip had a lower risk of fracture than women in the placebo group who had a $4 \%$ gain in bone mineral density at this site. ${ }^{7}$ How much these findings reflect variation in the measurement of bone mineral density or a true lack of correlation between changes in bone mineral density and fracture reduction is uncertain, but the clear implication for clinical practice is that patients may be given inappropriate advice if changes in bone mineral density are used to monitor treatment. example, only $16 \%$ and $4 \%$ of the decrease in the risk 
Because adherence to treatment for osteoporosis is poor, it could be argued that monitoring bone mineral density is justified because it should improve adherence and thereby maximise efficacy. We have no evidence to support this, however, and it would be difficult to identify people who do not adhere to this test because of the large measurement variability. Because non-adherence occurs mostly within the first three months of starting treatment, early intervention would probably be more effective, and evidence exists that an interview with a healthcare professional a few months after starting treatment improves adherence..$^{11}$ Biochemical markers of bone turnover could potentially be used for monitoring because they change rapidly in response to treatment and are more predictive of fracture reduction. However, at present within person variability and measurement variability are too great for these markers to be useful in clinical practice. ${ }^{12}$

If true non-responders to antiresorptive treatment do exist they are rare, and most cases of non-response are caused by failure to persist with treatment. This is best tackled by carefully explaining the treatment to patients before they start and follow-up after about three months to discuss problems related to treatment. Routine monitoring of bone mineral density during the first few years of antiresorptive treatment cannot be justified because it may mislead patients, lead to inappropriate management decisions, and waste scarce healthcare resources.
1 International Society for Clinical Densitometry (ISCD). Official positions 2007.www.iscd.org/Visitors/positions/OP-Index.cfm.

2 National Osteoporosis Foundation (NOF). New clinician's guide to prevention and treatment of osteoporosis. 2008. www.nof.org/ professionals/Clinicians Guide.htm.

3 Leslie WD. Factors affecting short-term bone density precision assessment and the effect on patient monitoring. J Bone Miner Res 2008;23:199-204

4 Lindsay R, Pack S, Li Z. Longitudinal progression of fracture prevalence through a population of postmenopausal women with osteoporosis. Osteoporos Int 2005;16:306-12.

5 Bell KJL, Hayen A, Macaskill P, Irwig L, Craig JC, Ensrud K, et al. Value of routine monitoring of bone mineral density after starting bisphosphonate treatment: secondary analysis of trial data BMJ 2009;338:b2266.

6 Cummings SR, Karpf DB, Harris F, Genant HK, Ensrud K, LaCroix AZ, et al. Improvement in spine bone density and reduction in risk of vertebral fractures during treatment with antiresorptive drugs. Am J Med 2002;112:281-9.

7 Sarkar S, Mitlak BH, Wong M, Stock JL, Black DM, Harper KD. Relationships between bone mineral density and incident vertebral fracture risk with raloxifene therapy. J Bone Miner Res 2002;17:1-10.

8 Watts NB, Geusens P, Barton IP, Felsenberg D. Relationship between changes in BMD and nonvertebral fracture incidence associated with risedronate: reduction in risk of nonvertebral fracture is not related to change in BMD. J Bone Miner Res 2005;20:2097-104.

9 Chapurlat RD, Palermo L, Ramsay P, Cummings SR. Risk of fracture among women who lose bone density during treatment with alendronate. The Fracture Intervention Trial. Osteoporos Int 2005; $16: 842-8$

10 Szulc P, Delmas PD. Biochemical markers of bone turnover: potential use in the investigation and management of osteoporosis. Osteoporos Int 2008;19:1683-704.

11 Compston J, Seeman E. Compliance with osteoporosis therapy is the weakest link. Lancet 2006;368:973-4.

12 Clowes IA, Peel NFA, Eastell R. The impact of monitoring on adherence and persistence with antiresorptive treatment for postmenopausal osteoporosis: a randomized controlled trial. J Clin Endocrinol Metab 2004;89:1117-23.

\section{Helen Roberts senior lecturer} women's health, Department of Obstetrics and Gynaecology, University of Auckland, Auckland, New Zealand

h.roberts@auckland.ac.nz Competing interests: None declared.

Provenance and peer review: Commissioned; not externally peer reviewed.

Cite this as: $B M J$ 2009;338:b2116 doi: 10.1136/bmj.b2116

\section{Reduced use of hormones and the drop in breast cancer The lowest dose of hormones for the least amount of time should be used for the relief of menopausal flushes}

Recent studies have shown falls in the incidence of breast cancer in Australia and the United Kingdom. ${ }^{12}$ This decline in hormone receptor positive tumours was found in post-menopausal women but not in premenopausal women, and has also been noted across much of Europe and the United States. ${ }^{13}$ Most of these reports have linked this declining diagnosis of breast cancer to a decrease in use of hormone replacement therapy (HRT) following publication of the women's health initiative study. The oestrogen plus progestogen arm of this study was halted in May 2002 because of an excess risk of breast cancer. ${ }^{4}$

A subsequent US study that examined trends of breast cancer incidence and HRT use within a screened population likewise found a fall in the incidence of breast cancer corresponding to a decrease in the use of HRT. ${ }^{5}$ The rapid decrease in breast cancer incidence reported in these studies suggests that withdrawal of hormone therapy leads to a regression of preclinical cancers. ${ }^{6}$

In the first 2 years of the oestrogen plus progestogen versus placebo arm of the women's health initiative study, fewer breast cancers were diagnosed in the women taking combined HRT than in those taking placebo. This finding is thought to be the result of increases in breast density induced by the hormones, making the mammograms harder to interpret and causing delay in diagnosis. Thereafter, the number of cases of breast cancer increased for the women on HRT (hazard ratio $1.26,95 \%$ CI 1.02 to 1.55 ), and the cancers being diagnosed were larger and more advanced than those in women not on HRT. ${ }^{4}$ After discontinuation, follow-up of the women who had stopped HRT showed that the raised risk of breast cancer in this group had decreased rapidly: the risk was $28 \%$ less in the first post-intervention year despite a similar frequency of mammography. ${ }^{6}$

The increased risk was mainly in women who had used oestrogen and progestogen before joining the study $(1.96,1.17$ to 3.27$)$, as the risk was considerably lower in those who had not used these hormones $(1.02,0.77$ to 1.35). There was, however, a significant and increasing trend in the risk of breast cancer with follow-up time among women with no previous hormone use. Ongoing use for durations only slightly longer than 5 years does seem to be associated with an increased risk of breast cancer in women who have not previously used hormones. ${ }^{4}$ The combined analysis of the clinical and 
observational women's health initiative studies also suggests that women who initiate oestrogen plus progestogen soon after menopause and continue for many years seem to be at particularly high risk of breast cancer. ${ }^{7}$

The combined analysis of the clinical and observational results also looked at the effect of "gap time"-that is, the time from menopause to first use of hormones. This analysis showed that the decreased risk of breast cancer associated with oestrogen therapy only occurred if the hormone was used later in the post-menopausal period and not

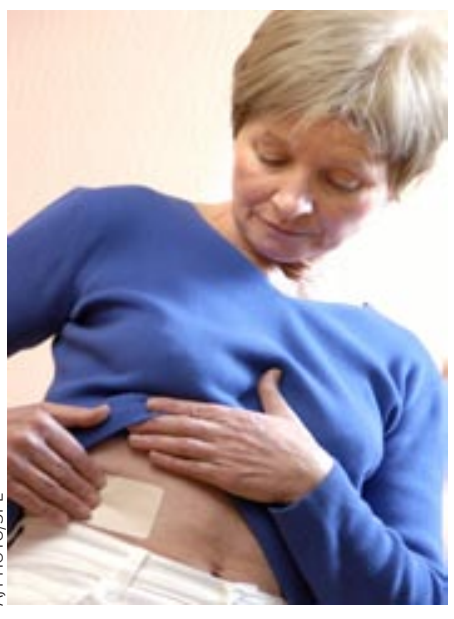

breast cancer according to the Gail model. ${ }^{8}$ Caution is therefore advised in prescribing hormone therapy to women who are already at high risk of breast cancer.

For women diagnosed with breast cancer chemotherapy, ovarian ablation, and adjuvant therapy can all contribute to the occurrence of severe flushes. Clinical trials comparing hormone replacement therapy and tibolone with placebo in women with previous breast cancer found increased breast cancer recurrence for those women using hormones. Hormone use following breast cancer treatment should thus be avoided. ${ }^{1011}$

Placebo controlled randomised trials have found that clonidine, gabapentin, selective serotonin reuptake inhibitors, and selective noradrenaline reuptake inhibitors can reduce hot flushes. The antidepressant drug venlaflaxine seems to interfere less than other antidepressants with the efficacy of tamoxifen. Vitamin $\mathrm{E}$ can be tried in women wishing to avoid taking pharmaceutical agents. ${ }^{12}$

These recent reports of a reduction in the incidence of breast cancer following a drop in hormone use, along with the results from ongoing analyses of the women's health initiative studies, support the use of the lowest dose of hormones for the shortest period of time for the relief of menopausal flushes. This approach will not only decrease the risk of hormone related breast cancers in the individual, but also the risk for entire populations.

1 Kumle M. Declining breast cancer incidence and decreased HRT use. Lancet 2008;372:608-10.

2 Parkin DM. Is the recent fall in incidence of post-menopausal breast cancer in UK related to changes in use of hormone replacement therapy? Eur J Cancer 2009, doi:10.1016/j.ejca.2009.01.016.

3 Jemal A, Ward E, Thun MJ. Recent trends in breast cancer incidence rates by age and tumor characteristics among US women. Breast Cancer Res Treat 2007;9:R28.

4 Anderson GL, Chlebowski RT, Rossouw JE, Rodabough RJ, McTiernan A, Margolis KL, et al. Prior hormone therapy and breast cancer risk in the Women's Health Initiative randomized trial of estrogen plus progestin. Maturitas 2006;55:103-15.

5 Kerlikowske K, Miglioretti DL, Buist DS, Walker R, Carney PA, National Cancer Institute-Sponsored Breast Cancer Surveillance Consortium. Declines in invasive breast cancer and use of postmenopausal hormone therapy in a screening mammography population. J Natl Cancer Inst 2007;99:1335-9.

6 Chlebowski RT, Kuller LH, Prentice RL, Stefanick ML, Manson JE, Gass M, et al. Breast cancer after use of estrogen plus progestin in postmenopausal women. N Engl J Med 2009;360:573-87.

7 Prentice RL, Chlebowski RT, Stefanick ML, Manson JE, Pettinger $M$, Hendrix SL, et al. Estrogen plus progestogen therapy and breast cancer risk in recently menopausal women. Am J Epidemiol 2008;167:1207-16.

8 Prentice RL, Chlebowski RT, Stefanick ML, Manson JE, Langer RD, Pettinger $\mathrm{M}$, et al. Conjugated equine estrogens and breast cancer risk in the women's health initiative clinical trial and observational study. Am J Epidemiol 2008;167:1407-15.

9 Chlebowski RT, Prentice RL. Menopausal hormone therapy in BRCA1 mutation carriers: uncertainty and caution. J Natl Cancer Inst 2008;100:1341-3.

10 Holmberg L, Anderson H. HABITS (hormone replacement therapy after breast cancer-is it safe?), a randomized comparison trial stopped. Lancet 2004;363:453-5.

11 Kenemans P, Bundred NJ, Foidart JM, Kubista E, von Schoultz B, Sismondi P, et al. Safety and efficacy of tibolone in breast cancer patients with vasomotor symptoms: a double blind, randomized, non-inferiority trial. Lancet Oncol 2009;10:135-46.

12 Roberts H. Managing the menopause. BMJ 2007;334:736-41. 